\title{
Video Article \\ A Computerized Functional Skills Assessment and Training Program Targeting Technology Based Everyday Functional Skills
}

\author{
Philip D. Harvey ${ }^{1,2}$, Lize Tibiriçá ${ }^{2,3}$, Peter Kallestrup ${ }^{2}$, Sara J. Czaja ${ }^{1,2,4}$ \\ ${ }^{1}$ University of Miami Miller School of Medicine \\ 2 i-Function \\ ${ }^{3}$ Albizu University \\ ${ }^{4}$ Weill-Cornell Medical Center
}

Correspondence to: Philip D. Harvey at pharvey@miami.edu

URL: https://www.jove.com/video/60330

DOI: doi: $10.3791 / 60330$

Keywords: Medicine, Issue 156, Functional Skills, Computerized Cognitive Training, Technology, Everyday Activities, Mild Cognitive Impairment, Aging

Date Published: 2/13/2020

Citation: Harvey, P.D., Tibiriçá, L., Kallestrup, P., Czaja, S.J. A Computerized Functional Skills Assessment and Training Program Targeting Technology Based Everyday Functional Skills. J. Vis. Exp. (156), e60330, doi:10.3791/60330 (2020).

\section{Abstract}

Today, many functional skills are technology-based, so development of a technology-based training program has broad importance. Here we present a computerized functional skills training program that was paired in half of the participants with a commercially available cognitive training (CCT) program.

Non-impaired older individuals $(\mathrm{NC})$ aged $60+(n=45)$ and similarly aged individuals with mild cognitive impairment $(\mathrm{MCl} ; n=50)$ were randomized to receive 12 weeks of twice-weekly computerized functional skills training (CFST) or 12 weeks of twice-weekly sessions split between CCT and CFST. Skills trained were use of an ATM; internet banking; ticket kiosk; telephone and internet prescription refill; medication management; and internet shopping. As with previous functional capacity assessments, we focus on completion time for each simulation.

51 participants completed the training program, either by mastering all 6 tasks (34) or completing 12 weeks of training. 44 more participants completed 4 or more training sessions so they were also analyzed for improvement up to their last training session. Completion time for all 6 tests significantly improved from the baseline assessment to the final training session in both groups of participants (all $p<0.001$ with an average improvement in task completion time of 45\%). Further, there was no differential improvement in $\mathrm{MCl}$ and $\mathrm{NC}$ in the 6 tests from baseline to end of training (all $\mathrm{t}<1.66$, all $\mathrm{p}>0.12$ ). Finally, combined CCT plus CFST did not differ from CSFT alone on any of the percent-change score measures (all $\mathrm{t}<1.64$, all $\mathrm{p}>0.11$ )

Both $\mathrm{NC}$ and $\mathrm{MCl}$ groups evidenced substantial improvements in performance. CCT supplementation led to similar functional gains with half as many training sessions. The NC participants proceeded through the training fairly rapidly even without CCT supplementation; $\mathrm{MCl}$ participants required more training but learned equivalently. These findings suggest that even in cases with memory impairments, functional skills can be efficiently learned with training.

\section{Video Link}

The video component of this article can be found at https://www.jove.com/video/60330/

\section{Introduction}

Many contemporary functional tasks are performed using technology. This includes banking and other financial management tasks, travel and transit tasks, and healthcare management. The challenges of using technology daily are amplified in older people whose lifetime exposure to technology may be more limited. Technology-based tasks can also be cognitively demanding. Older people and people with different challenges, such as severe mental illness ${ }^{1,2}$ or cognitive deficits, may not have the financial or cognitive resources to use challenging technologies. These individuals have difficulty negotiating today's digitized world, which is a threat to their independence.

Our previous research has shown that many healthy older people also have problems in performing everyday functional tasks efficiently ${ }^{3}$. Further, people with mild cognitive impairment have proportionately greater challenges performing these tasks ${ }^{4}$. Our research has shown that component cognitive abilities in healthy aging ${ }^{4}$, severe mental illness ${ }^{5}$ and $\mathrm{MCl}$ populations, is consistently correlated with the ability to perform veridical simulations of everyday functional tasks. Thus, cognitive ability is a rate limiter for people's ability to initially perform, and learn, functional tasks using technology. This problem is exacerbated by the fact that many of these tasks, such as using an ATM, are thought of as "walk up tasks" or tasks that are intuitive and do not require training.

Currently, training in the performance of technology-relevant everyday functional is not systematically delivered. New technology, such as iPhones, tablets, and computers, are commonly delivered without any instructions for their use. Instructions for using websites are not commonly 
available. For example, the New York Metropolitan Transit Authority (MTA), website has no instructions on using their ticket kiosks other than instructions on how to refill a Metrocard.

The incremental deficits in cognitive performance can be partially addressed with computerized cognitive training (CCT) for some populations. Recent data have suggested that both healthy older people ${ }^{6}$ and people with $\mathrm{MCl}^{7}$ can make cognitive gains with CCT on the cognitive abilities targeted by the training. Thus, it would be expected that performance of functional tasks would also be facilitated with CCT efforts. However, the widely reported success of CCT in both older adult and $\mathrm{MCl}$ populations has not been accompanied by spontaneous improvements in the ability to perform novel functional skills. While previously acquired skills, such as driving ${ }^{8}$, can be facilitated through CCT, there is no evidence across populations that CCT alone is enough to lead to acquisition of novel functional skills.

CCT has also been shown to have protective effects against development of dementia, at least with a limited set of training routines. For instance, the ACTIVE trial showed that computerized speed training was associated with persistent improvements in cognitive performance that could be detected at a 10 -year follow-up ${ }^{9}$. A subsequent follow-up study also reported a $30 \%$ decrease in dementia associated with all causes at the 10 -year period as well ${ }^{10}$. As a result, since certain types of CCT appear to result in cognitive benefits among older people, the combination of CCT and computerized functional skills training should result in improvements in functional skills.

Thus, the current program involved the development of a set of ecologically valid functional skills tasks that are typically performed using some sort of technology, either internet, telephone, or in-person on a device such as an ATM. The tasks are presented in Table 1 and were chosen as important to living independently. Within the program, these tasks are performed in fixed difficulty, non-training formats first. Each task has multiple different graduated sub-tasks, which vary in difficulty of functional demands. After completion of the 6 fixed difficulty tasks, all participants are then trained on the Computerized Functional Skills Training (CFST) training simulations. These simulations provide direct performance feedback to the participant. Sample feedback is presented in Table 2. There is no involvement of a human trainer, nor does a human provide feedback. When a participant makes an error on a subtask, graduated feedback is provided across that increases in corrective information. For example, in the ATM task, if an individual makes an initial error inputting their pin number, basic corrective information is provided; if they make the same error a second time more corrective information is provided.

After 4 errors, the task proceeds to the next training step. However, when the participant returns to train later, this step is re-trained until it is passed. Each of the training modules is considered complete and the participant graduates after performing the entire task two times without any errors.

The study includes two research participant groups: (1) cognitively normal (CN) healthy older and (2) medically healthy older people with mild cognitive impairment (MCI). CN was defined as a Montreal Cognitive Assessment (MOCA $)^{11}$ score of 26 or more and no cognitive complaints. $\mathrm{MCl}$ was defined with a systematic assessment that included the MOCA, assessments of subjective complaints, and assessment with a structured neuropsychological assessments. Participants were excluded if their cognitive performance reflected impairment greater than MCI. Training was conducted on Windows computers although the software can be deployed in iOS as well. Training was proctored in a ratio of approximately 6 trainees per proctor.

The goal of the study is to determine 1) if CFST is efficacious in healthy older people, defined by improvements in performance of computerbased functional skills; (2) the relative efficacy of functional skills training for those with $\mathrm{MCl}$ as compared to those who are non-impaired; and (3) whether the provision of CCT enhances the CFST and if there is a differential effect for the $\mathrm{MCl}$ as compared to the NC.

\section{Protocol}

The study is a randomized trial, where half of the research participants (stratified by cognitive status) are randomized 1 to 1 to receive computerized cognitive training (CCT) on the Double-Decision task from the Posit Science Brain HQ training software combined with the CFST and the others are trained on the CFST alone. This research was reviewed and approved by the University of Miami Institutional Review Board and all participants provided signed informed consent.

\section{Preparation}

1. Obtain written informed consent.

2. Screen the participant with the Montreal Cognitive Assessment (MOCA) ${ }^{11}$.

1. Have the participant connect numbers and letters in sequence.

2. Show the participant 3 pictures of animals and have them name them.

3. Read 3 verbal learning words and have participant recall them.

3. Perform a baseline cognitive assessment using the tablet version of the Brief Assessment of Cognition (Figure 1) $)^{12}$. Administer all of the subtests on the tablet app. The subtests are Verbal Learning and Memory, Digit Sequencing, Token Motor Task, Symbol Coding, Verbal Fluency Examinations, and Tower of London.

1. Present the overall instructions for the assessment on the app.

2. Present the instructions for the Symbol Coding Task.

3. Have the participant do practice coding.

4. Watch the participant perform the coding task for 15 seconds.

5. Present the instructions for the Tower of London Task.

6. Have the participant do the practice items.

7. Watch the participant solve the first test item.

4. Assess the participant with 6 different functional tasks (Figure 2) in a fixed difficulty format. 
NOTE: During the fixed difficulty assessment, there is no training provided, and if a participant makes an error, the instructions are simply repeated. When a participant completes a task, they proceed to the next one. When all of the tasks are completed, training begins. The representative task demands of each task are presented below.

\section{Task demands for fixed difficulty and training simulations}

1. Launch the Ticket Purchase Task.

1. Select purchase a new ticket.

2. Select Purchase a single ride ticket.

3. Check the balance on the transit card.

4. Add $\$ 60.00$ to the transit card.

2. Launch the telephone refill task.

1. Dial the number for the pharmacy.

2. Enter the prescription number.

3. Select the time to pick up the medication.

3. Launch the ATM banking task.

1. Enter the PIN to start the session.

2. Check the balance in the checking account.

3. Withdraw $\$ 180.00$ from the checking account

4. Launch the medication label comprehension task.

1. Choose the correct time of day to take the medication.

2. Correctly identify how many pills to take each day.

3. Launch the medication organizer subtask.

4. Pack up a day's worth of medications.

5. Launch the internet banking task.

1. Enter the User ID and password.

2. Check the balance in the checking account.

3. Make a transfer of $\$ 15.00$ from savings to checking.

6. Launch the internet prescription refill and online shopping task.

1. Enter the userID and password.

2. Verify your identity by selecting the correct city (Miami), Street (Micapony) and Car color (Blue).

3. Select Prinivil and no generic equivalent.

4. Select Correct date and time for pickup.

\section{Training after the fixed difficulty assessment}

1. CFST alone condition

1. Explain the training task to the participant, stating that all 6 tasks will be trained.

2. Launch the ATM banking task.

3. Enter PIN.

4. Select transaction (check balance in checking).

5. Visualize the process of program-delivered feedback for errors.

6. Present 4 errors in sequence to demonstrate the feedback process.

2. CCT + CFST Condition

NOTE: The CFST training is the same as CFST alone but is for 30 minutes only. Brain HQ DoubleDecision is the computerized cognitive training task in the combined condition. Figure 3 depicts the Double-Decision Task.

1. Explain the process of Training on Computerized cognitive training as well as skills training.

2. Launch Brain HQ double Decision Task.

3. Perform the practice items.

4. Perform the actual test items.

5. Visualize this process for about 30 seconds.

\section{Post-training assessment}

NOTE: After graduation from all six training tasks or completion of 24 sessions, participants complete a post-test. This next section should be presented as a verbal description of the post training assessments

1. Have participants perform different versions of all six trained tasks. They will be told that the tasks are the same, but the content demands are different.

2. Have participants perform the BAC App assessment again. 


\section{Representative Results}

The patient flow is shown in Table 3. The first subject was screened on July 14, 2018. Screened participants included 78 females and 76 males, of whom $53(33 \%)$ were Latino and $52(33 \%)$ are of African American descent. Mean levels of educational attainment were similar across ethnic groups and averaged 15 years. However, there were cases who were training who had less than 8 years of education. 131 cases completed the eligibility assessment, with 16 cases excluded because of possible dementia and 4 cases excluded because of motor or vision problems. Of those 121 randomized $46 \%(n=56)$ were $\mathrm{HC}$ and $54 \%(n=65)$ were diagnosed at $\mathrm{MCl}$ and $50 \%$ in each group was randomized to CFST training alone. 51 cases completed training with 34 of them graduating, which translates into perfect performance twice in sequence on 30 individual subtasks. 44 cases are still training and 15 cases are waiting to train. The drop-out rate after any training was $9 \%$. The mean MOCA score was $28.38(S D=1.70)$ for the NC group and $22.68(S D=3.02)$ for the $\mathrm{MCl}$ group.

Figure 4 presents the training results for completers and Figure 5 presents the results for all participants who trained. These data are presented in terms of time to completion, although several other dependent variables are collected. Paired t-tests found that completion time for all 6 tests significantly improved from the baseline assessment to the final training assessment in completers (all $t>8.16$, all $p<.001)$. Further, none of the simulations improved differentially in the $\mathrm{NC}$ and $\mathrm{MCl}$ samples, as indexed by percentage of improvement from baseline to end of training, all $t<1.66$, all $p>$.12. Finally, combined CCT plus CFSAT did not differ from CSFAT alone on any of the \%-change score measures: All $t<1.44$, all p>.16. CCT supplementation led to similar CFSAT gains with half as many CFSAT training sessions and this improvement was consistent in the $\mathrm{MCl}$ and $\mathrm{NC}$ groups in both conditions. All t-tests for the six tests, across the $\mathrm{MCl}$ vs. NC samples and the skills training only vs. combined assessments are in Table 4. Both groups evidenced substantial improvements in performance on alternative versions of the assessment tasks. Importantly, NC participants who received skills training alone required an average of only 6 trainings per module to perfect their performance. The participants randomized to CCT \& CFSAT trained an average number of 11 days on CCT, achieving an average of 50 levels per participant.

BAC tower of London

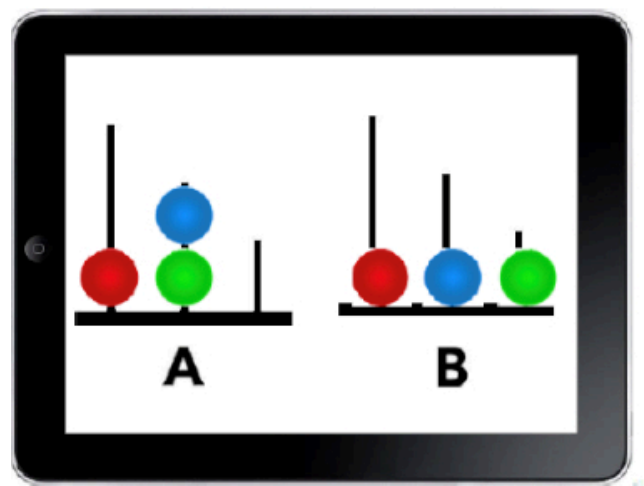

BAC Symbol Coding

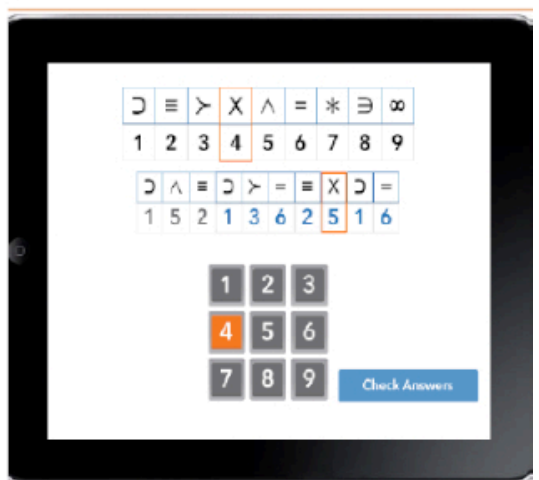

Figure 1: Representative stimuli from the BAC App showing the tower of London and Symbol coding subtests. Please click here to view a larger version of this figure.

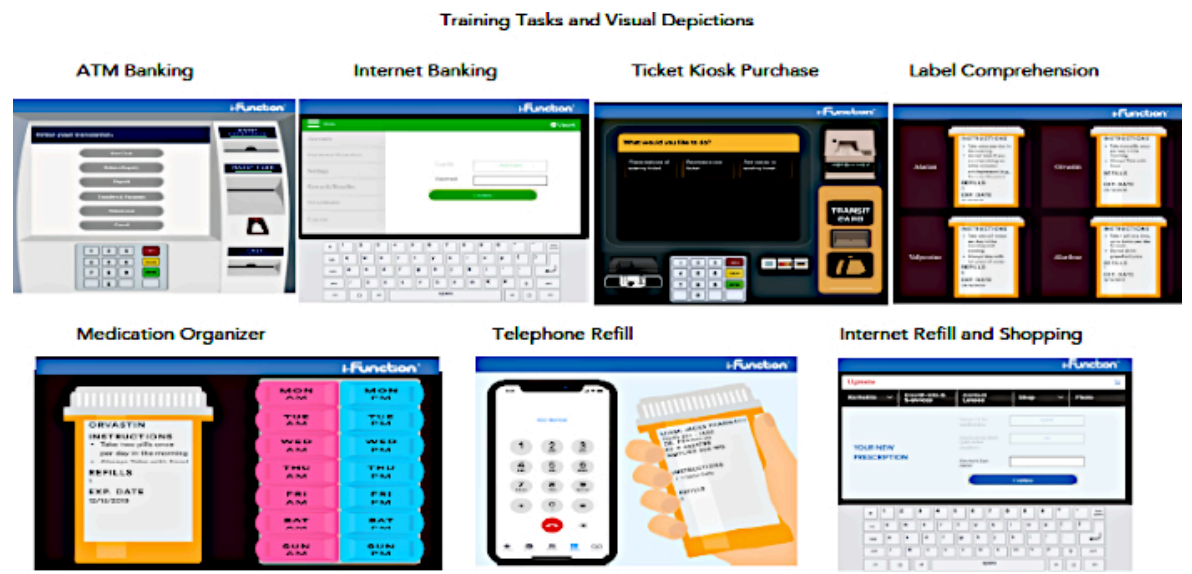

Figure 2: The 6 CFST training tasks. These tests include Ticket Purchase, ATM and Internet Banking, Phone and Internet Prescription Refill and Shopping, and Medication Management Please click here to view a larger version of this figure. 


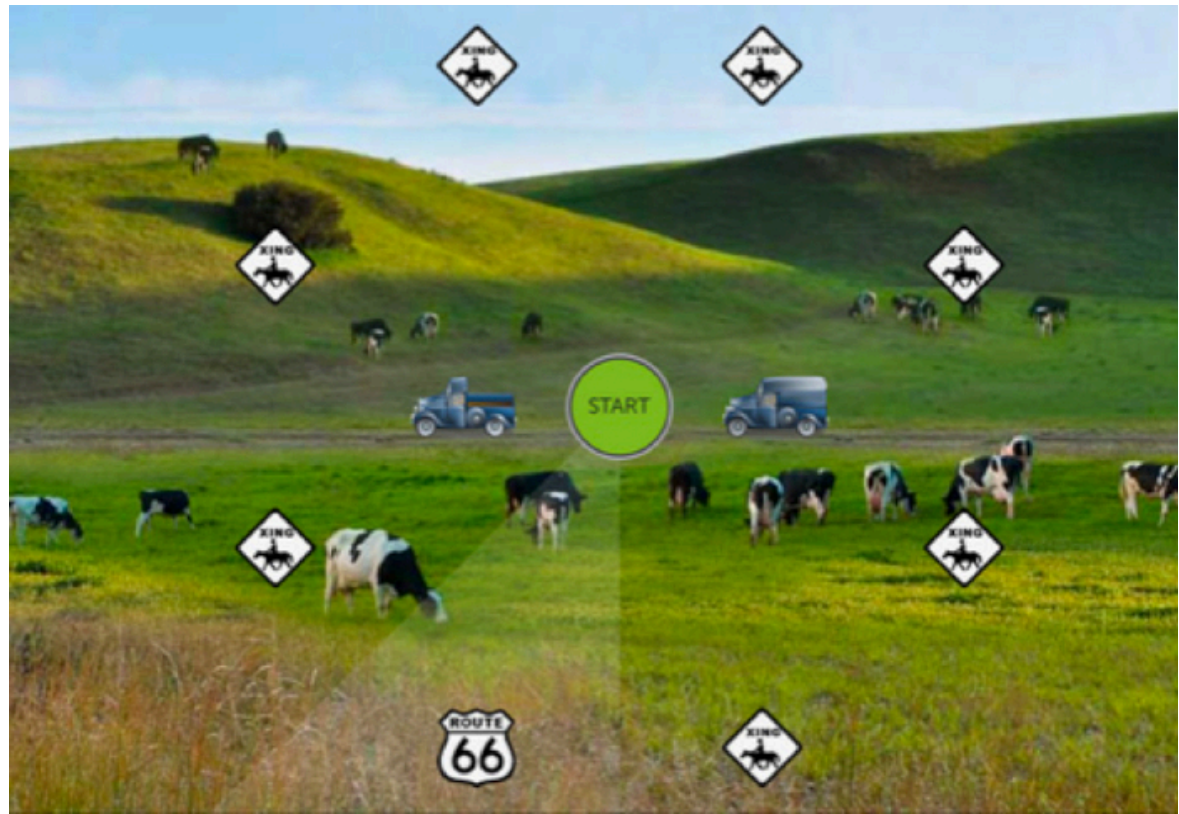

Figure 3: Brain HQ Double Decision Task Stimulus. Please click here to view a larger version of this figure.

Time to Completion for All Six Training Tasks at Baseline, end of Training, and Posttest: Completers

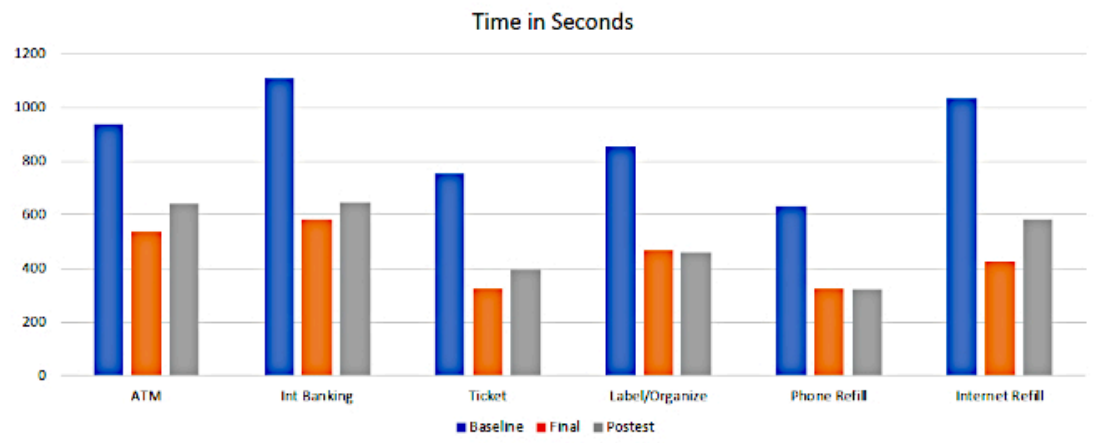

Note: $n=51$

Figure 4: Training gains from baseline in individuals who completed training. This is depicted in terms of time to completion at the first assessment, the final training session, and the alternate form of the simulations, across the 6 training tasks. Please click here to view a larger version of this figure. 


\section{Percentage Gain Per training Session: Completers and Still Training}

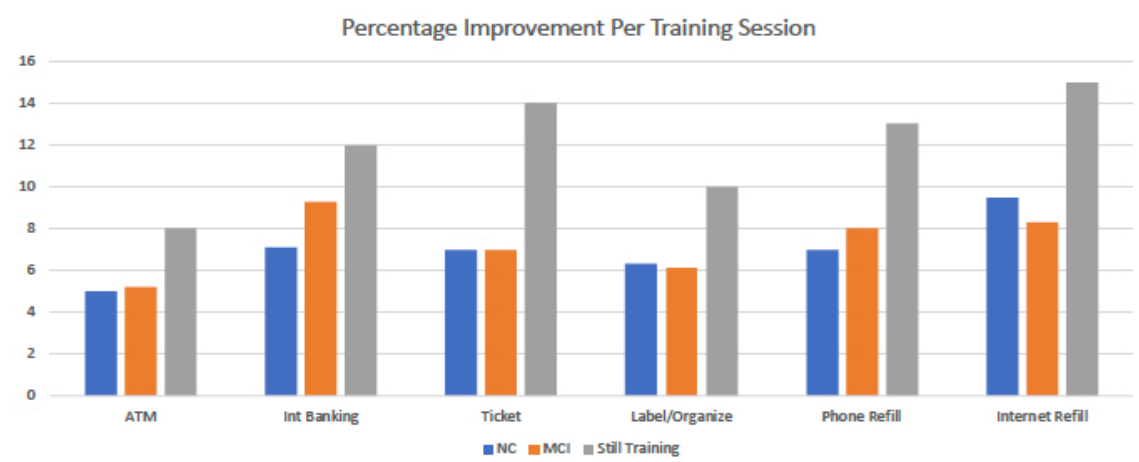

Note. $\mathrm{NC}=36, \mathrm{MCl}=15$, Still Training=44

Figure 5: Percentage improvement per training session for $\mathrm{NC}$ and $\mathrm{MCl}$ completers and cases still training. This is depicted in terms of the proportion of the total baseline to last session training gains in time to completion per training session completed. Please click here to view a larger version of this figure.

\begin{tabular}{|l|}
\hline ATM Banking \\
\hline Internet Banking \\
\hline Ticket Purchase with Kiosk \\
\hline Medication Management and Daily Organizer \\
\hline Interactive Telephone Voice Menu for Prescription Refill \\
\hline Internet Prescription Refill and On-Line Shopping \\
\hline
\end{tabular}

\section{Table 1: Computerized functional skills training (CFST) tasks}

\begin{tabular}{|l|l|}
\hline Error 1 Repeat Instruction: & Your Pin is 1234. Please enter your PIN \\
\hline Error 2. Guidance: & Your PIN is 1234. Please use the keypad to enter your PIN \\
\hline Error 3 Direction: & Your PIN is 1234. Enter 1, followed by 2, followed by 3, followed by 4 \\
\hline Error 4 Demonstration & $\begin{array}{l}\text { The four keys are lit up in sequence and the participants is instructed to } \\
\text { touch them when they light up. }\end{array}$ \\
\hline
\end{tabular}

Table 2: Error feedback.

\begin{tabular}{|l|l|l|}
\hline Participant Flow in Clinical Trial (July 2018 to Present) & \\
\hline Screened and signed consent & 154 & \\
\hline Ineligible & 20 & \\
\hline Withdrew before baseline & 4 & \\
\hline Baseline not completed & 9 & \\
\hline Completed baseline assessment & 121 & $42 \%$ \\
\hline Completed training & 51 & $36 \%$ \\
\hline Still training & 44 & $12 \%$ \\
\hline Waiting to train & 15 & $9 \%$ \\
\hline Dropped out after training & 11 & \\
\hline
\end{tabular}

Table 3: The CONSORT Diagram for the clinical trial. 


\begin{tabular}{|c|c|c|c|}
\hline \multicolumn{2}{|l|}{ ATM Banking } & \multirow{2}{*}{\begin{tabular}{|l|}
$t$ \\
0.98
\end{tabular}} & \multirow{2}{*}{$\frac{p}{0.33}$} \\
\hline & $\mathrm{HC} \mathrm{VS} \mathrm{MCl}$ & & \\
\hline & Skills only vs. Combined Training & 0.86 & 0.4 \\
\hline \multicolumn{4}{|c|}{ Medication Management } \\
\hline & $\mathrm{HC} \mathrm{VS} \mathrm{MCl}$ & 0.57 & 0.57 \\
\hline & Skills only vs Combined Training & 0.91 & 0.37 \\
\hline \multicolumn{4}{|c|}{ On-Line Banking } \\
\hline & $\mathrm{NC}$ vs. $\mathrm{MCl}$ & 1.66 & 0.12 \\
\hline & Skills only vs Combined Training & 0.56 & 0.96 \\
\hline \multicolumn{4}{|c|}{ Prescription Refill } \\
\hline & $\mathrm{NC}$ vs. $\mathrm{MCl}$ & 0.21 & 0.84 \\
\hline & Skills only vs. Combined Training & 1.44 & 0.16 \\
\hline \multicolumn{4}{|c|}{ Ticket Purchase Task } \\
\hline & $\mathrm{NC}$ vs. $\mathrm{MCl}$ & 1.25 & 0.22 \\
\hline & Skills only vs. Combined Training & 0.25 & 0.81 \\
\hline \multicolumn{4}{|c|}{ Internet Prescription Refill and Shopping } \\
\hline & $\mathrm{NC}$ vs. $\mathrm{MCl}$ & 1.55 & 0.19 \\
\hline & Skills Only vs. Combined Training & 0.16 & 0.87 \\
\hline
\end{tabular}

Table 4: Results of t-tests comparing $\mathrm{MCl}$ vs. NC training gains and Skills only vs. Combined Treatment.

\section{Discussion}

CSFT training led to substantial and rapid treatment gains with as few as 6 training sessions, with results applicable to both NC and MCI participants. Both participant groups evidenced substantial improvements in task performance. CCT supplementation led to similar CFST gains with half as many CFST training sessions. Importantly, NC participants who received skills training alone required an average of only 6 sessions per task (out of a possible 24) to perfect their performance. In summary: 1) both groups of participants demonstrated improvements in performance across all tasks; 2 ) the $\mathrm{HC}$ participants proceeded through the training fairly rapidly even without CCT supplementation; and 3 ) $\mathrm{MCl}$ participants required more training sessions but learned equivalently. These findings replicate our previous findings with older patients with schizophrenia and a separate sample of healthy controls.

Of primary importance is the improvement in training associated with computerized functional skills training in participants with MCl. These cases had substantial impairments in their episodic memory. However, they were still able to make substantial gains, proportionately equivalent to people with NC, across 6 different training simulations. Previous studies have shown dissociation of procedural and verbal memory learning in $\mathrm{MCl}$ and amnestic conditions ${ }^{13,14}$. Thus, this study shows that functional skills can be learned fairly rapidly and efficiently, with few dropouts.

Supplementation of computerized skills training with CCT increased the efficiency of the skills training considerably, with gains doubled per unit training session compared to skills training alone. Thus, a combined intervention with CCT and CFST in individuals with MCI would likely have multiple benefits. First, dementia prevention may be facilitated by CCT. Skills training may also lead to increased independence or delay progressive functional changes in $\mathrm{MCl}$. As a result, the potential benefits of combined training seem considerable and a major topic for further study with this protocol.

Later studies will focus on real-world functional gains. Demonstration of such real-world gains would solidify the benefits of this training protocol. Implementing the protocol was quite efficient and participants reported high levels of satisfaction with their gains. For example, $98 \%$ or more of the participants stated that they "definitely" would be more able to do each of the 6 tasks in the real world.

\section{Disclosures}

In the past year Dr. Harvey has received consulting fees or travel reimbursements from Alkermes, Boehringer Ingelheim, Intra-Cellular Therapies, Jazz Pharma, Minerva Pharma, Otsuka America, Roche Pharma, Sanofi Pharma, Sunovion Pharma, Takeda Pharma, and Teva. He receives royalties from the Brief Assessment of Cognition in Schizophrenia. He is Chief Scientific Officer for i-Function. Peter Kallestrup is CEO of i-Function. Lize Tibiriçá is a part time employee of i-Function. Sara Czaja is Chief Scientific officer of i-Function.

\section{Acknowledgments}

This research was funded by NIA grant number R43AG057238 to Peter Kallestrup. 


\section{References}

1. Berkowsky, R.W., Sharit, J., Czaja, S.J. Factors Predicting Decisions About Technology Adoption Among Older Adults. Innovations in Aging. 2 (1) (2018).

2. Harvey, P.D., Keefe, R.S.E. Technology, society, and mental illness: challenges and opportunities for assessment and treatment. Innovations in Clinical Neuroscience. 9, 47-50 (2012).

3. Taha, J., Czaja, S.J., Sharit, J., Morrow, D.G. Factors affecting usage of a personal health record (PHR) to manage health. Psychology and Aging. 28, 1124-1139 (2013).

4. Czaja, S.J., Loewenstein, D.A., Sabbag, S.A., Curiel, R.E., Crocco, E., Harvey, P.D. A Novel Method for Direct Assessment of Everyday Competence Among Older Adults. Journal of Alzheimers Disease. 57 (4), 1229-1238 (2017).

5. Czaja, S.J., Loewenstein, D.A., Lee, C.C., Fu, S.H., Harvey, P.D. Assessing functional performance using computer-based simulations of everyday activities. Schizophrenia Research. 183, 130-136 (2017).

6. Harvey, P.D., McGurk, S.R., Mahncke, H., Wykes T. Controversies in computerized cognitive training.Biological Psychiatry: Cognitive Neuroscience and Neuroimaging. 3, 907-915 (2018).

7. Sherman, D.S., Mauser, J., Nuno, M., Sherzai, D. The Efficacy of Cognitive Intervention in Mild Cognitive Impairment (MCI): a Meta-Analysis of Outcomes on Neuropsychological Measures. Neuropsychology Review. 27, 440-484 (2017).

8. Edwards, J.D., Delahunt, P.B., Mahncke, H.W. Cognitive speed of processing training delays driving cessation. Journal of Gerontology. 64 , 1262-1267 (2009).

9. Rebok, G.W. et al. Ten-year effects of the Advanced Cognitive Training for Independent and Vital Elderly cognitive training trial on cognition and everyday functioning in older adults. Journal of the American Geriatrics Society. 62, 16-24. (2014).

10. Edwards, J. et al. Speed of processing training results in lower risk of dementia. Alzheimers Dementia. 3, 603-611 (2017).

11. Nasreddine, Z.S. et al. The Montreal cognitive assessment, MoCA: a brief screening tool for mild cognitive impairment. Journal of the American Geriatrics Society. 53, 695-699 (2005).

12. Atkins, A.S. et al. Validation of the tablet-administered Brief Assessment of Cognition (BAC App). Schizophrenia Research. 181, 100-106 (2017).

13. Gobel, E.W. et al. Implicit perceptual-motor skill learning in mild cognitive impairment and Parkinson's disease. Neuropsychology. 27 (3), 314-321 (2013).

14. Oudman, E., Nijboer, T.C., Postma, A., Wijnia, J.W., Van der Stigchel, S. Procedural Learning and Memory Rehabilitation in Korsakoff's Syndrome - a Review of the Literature. Neuropsychology Review. 25 (2), 134-148 (2015). 